\title{
Aerospace laser communications technology as enabler for worldwide quantum key distribution
}

\author{
Florian Moll*a , Harald Weinfurter ${ }^{\mathrm{b}}$, Markus Rau ${ }^{\mathrm{b}}$, Christopher Schmidt ${ }^{\mathrm{a}}$, Gwen Melén ${ }^{\mathrm{b}}$, Tobias \\ Vogl $^{\mathrm{b}}$, Sebastian Nauerth ${ }^{\mathrm{c}}$, Christian Fuchs ${ }^{\mathrm{a}}$ \\ ${ }^{a}$ German Aerospace Center (DLR), Institute of Communications and Navigation, 82234 Wessling, \\ Germany; bLU München, Faculty of Physics, 80799 Munich, Germany, ' qutools GmbH, 81371 \\ Munich, Germany.
}

\begin{abstract}
A worldwide growing interest in fast and secure data communications pushes technology development along two lines. While fast communications can be realized using laser communications in fiber and free-space, inherently secure communications can be achieved using quantum key distribution (QKD). By combining both technologies in a single device, many synergies can be exploited, therefore reducing size, weight and power of future systems. In recent experiments we demonstrated quantum communications over large distances as well as between an aircraft and a ground station which proved the feasibility of QKD between moving partners. Satellites thus may be used as trusted nodes in combination with QKD receiver stations on ground, thereby enabling fast and secure communications on a global scale. We discuss the previous experiment with emphasis on necessary developments to be done and corresponding ongoing research work of German Aerospace Center (DLR) and Ludwig Maximilians University Munich (LMU).

DLR is performing research on satellite and ground terminals for the high-rate laser communication component, which are enabling technologies for the QKD link. We describe the concept and hardware of three generations of OSIRIS (Optical High Speed Infrared Link System) laser communication terminals for low Earth orbiting satellites. The first type applies laser beam pointing solely based on classical satellite control, the second uses an optical feedback to the satellite bus and the third, currently being in design phase, comprises of a special coarse pointing assembly to control beam direction independent of satellite orientation. Ongoing work also targets optical terminals for CubeSats. A further increase of beam pointing accuracy can be achieved with a fine pointing assembly. Two ground stations will be available for future testing, an advanced stationary ground station and a transportable ground station. In parallel the LMU QKD source size will be reduced by more than an order of magnitude thereby simplifying its integration into future free-space optical communication links with CubeSats.
\end{abstract}

Keywords: Free-space optical communications, quantum key distribution, low earth orbit, CubeSat, air-ground experiment

\section{INTRODUCTION}

Fast and secure data communications is a matter of growing interest. Fast data communication may be handled by freespace optical communications technologies. These may be based on fiber or free-space propagation. Secure communications may be handled by quantum key distribution (QKD). Since both technologies exploit transmission of photons to fulfill the corresponding task, the combination of both functionalities in a single device bears high synergies, which decreases overall system complexity and cost. Current fiber based QKD is limited in distance because of fiber attenuation. Reported ranges go up to $307 \mathrm{~km}$ [1]. Even with incremental higher ranges, no key transmission over the full span of the European continent neither between continents seems to be possible in future. Fiber attenuation and detector noise will be the limiting factors. The fiber-given range limitation can be overcome by using satellite systems as trusted key nodes. Several research bodies all over the world work on exploiting QKD enabled by free-space optical (FSO) space communication systems to supply worldwide secure communications and different ideas exist on how to design a wide-span QKD system involving LEO and GEO satellites with receiver stations either in space or on ground [2-7].

*florian.moll@dlr.de; phone 498153 28-2876; fax 498153 28-2844; dlr.de

Copyright 2016 Society of Photo-Optical Instrumentation Engineers. One print or electronic copy may be made for personal use only. Systematic reproduction and distribution, duplication of any material in this paper for a fee or for commerical purposes, or modification of the content of the paper are prohibited.

Quantum Optics, edited by Jürgen Stuhler, Andrew J. Shields, Proc. of SPIE Vol. 9900

99000K - @ 2016 SPIE · CCC code: 0277-786X/16/\$18 - doi: 10.1117/12.2230232 
A range of experiments was already conducted to prove feasibility of QKD in space by performing quantum communications over long distances between aircraft and ground, balloon and ground and with space-borne sources and retroreflectors [8-12].

Generally, free-space optical communications enable high speed data transmission between moving partners over long distances which makes it attractive for satellite and aeronautical communications. Satellite applications involve communications between ground, low Earth orbit (LEO), geostationary orbit (GEO) and deep space to transmit various content like Earth observation-, science- or media data. Earth observation data recorded in LEO may be directly downlinked to ground or relayed via a geostationary node. The first case is covered by DLR's OSIRIS (Optical High Speed Infrared Link System). This will be, among other carriers, installed on the BiROS (Bispectral Infrared Optical System) satellite and will send the data from the thermography payload to ground [13]. The relay case is covered by the European Data Relay System. LEO Satellite payload data is send via laser link to a GEO satellite which relays the data via radio frequency to ground [14]. Science data which is gathered by deep space spacecraft can also be downlinked to Earth directly. The feasibility was shown with NASA's Lunar Atmosphere and Dust Environment Explorer which carried a laser communications payload for testing [15]. Media data shall be transmitted by GEO feeder links with extremely high bandwidth in the Terabit-per-second regime to supply, e.g., TV, radio and internet content [16]. Aeronautical applications involve connections from air to ground, air to space and air to air. Surveillance and observation missions can use, similar to the LEO case, direct downlinks or use a GEO as relay. The feasibility of airGEO links was demonstrated within the LOLA project [17]. Direct optical air to ground links were demonstrated for data download from airborne sensors in the former DLR projects Argos [18] and DODfast [19]. Free-space optical links are also considered for aeronautical networks by exploiting multiple air to air FSO and radio frequency links as investigated in [20]. These experiments and technology demonstrations prove the maturity of FSO communications for use in space and aeronautics. Except for feasibility analyses, demonstrations of QKD including space-borne quantum transmitters or receivers are still missing. This gap must be filled by upcoming missions and experiments.

The paper is structured as follows: the collaboration in the field of aeronautical quantum key distribution of DLR and LMU is reviewed in chapter 2, a demonstration of quantum key distribution involving an aircraft and a ground station as precursor for satellite systems as reported in detail in [9], [21]. The former and current works of DLR on optical satellite terminals and ground stations which can serve as QKD platforms are briefly described in chapter 3 . The family of OSIRIS terminals and IKN optical ground stations are considered as enabling technology for space QKD. The new work on quantum key distribution transmitters of LMU is outlined in chapter 4. An outlook for outstanding tasks and planned developments is given in chapter 5 .

\section{EXPERIMENTAL PROOF OF FEASIBILITY}

\subsection{QKD system}

Quantum key distribution [22] provides a new level of security for communication not possible for any conventional system based on the exchange of classical signals, and inherently belongs to the post-quantum crypto methods urgently required in the future. As the name indicates, it is not a new cryptography protocol but rather a way to securely distribute a key between two users, typically called Alice and Bob. According the first protocol proposed by Bennett and Brassard in 1984 (BB84) any eavesdropper's information about this key can be quantified, and consequently made arbitrarily small by shrinking the key, if one transmits non-orthogonal quantum states followed by post-processing over a classical communication channel. As the carrier of the quantum states, photons are the best choice. Advanced add-on protocols to BB84 also allow the usage of strongly attenuated light pulses simplifying the experimental implementation significantly. For free space channels the polarization of light is the most robust degree of freedom to manipulate. According to the initial BB84 scheme horizontal $(\mathrm{H}),+45^{\circ}(\mathrm{P})$, vertical $(\mathrm{V})$, and $-45^{\circ}(\mathrm{M})$ linear polarization can be used for encoding the quantum signals.

For the design of the QKD-sender module (Figure 1, left) we thus employ laser diodes to generate short pulses which are attenuated below the single photon level before the output of the module. The modulation of the polarization is one option, which however can be replaced by simply overlapping the light from four diodes. Each of them produces a different polarization direction and only one of them is activated at a time. Care has to be taken, that the properties of the light pulses generated by the four diodes, i.e., wavelength and timing, have to be indistinguishable to avoid side channels. In that sense, the design employs four laser diodes, oriented along horizontal or vertical direction, whose light is overlapped pairwise on two polarizing beam-splitters (PBS). After one of the PBS, a halve-wave plate is used to rotate 
the polarization in this arm from $\mathrm{H} / \mathrm{V}$ to $+45^{\circ} /-45^{\circ}$. Finally, the two beams with the four polarizations are overlapped on a polarization insensitive beam-splitter (BS). A spatial filter erases any residual path information and coupling mirrors provide alignment possibilities to overlap the quantum channel with the classical communication channel at a dichroic beam-splitter.
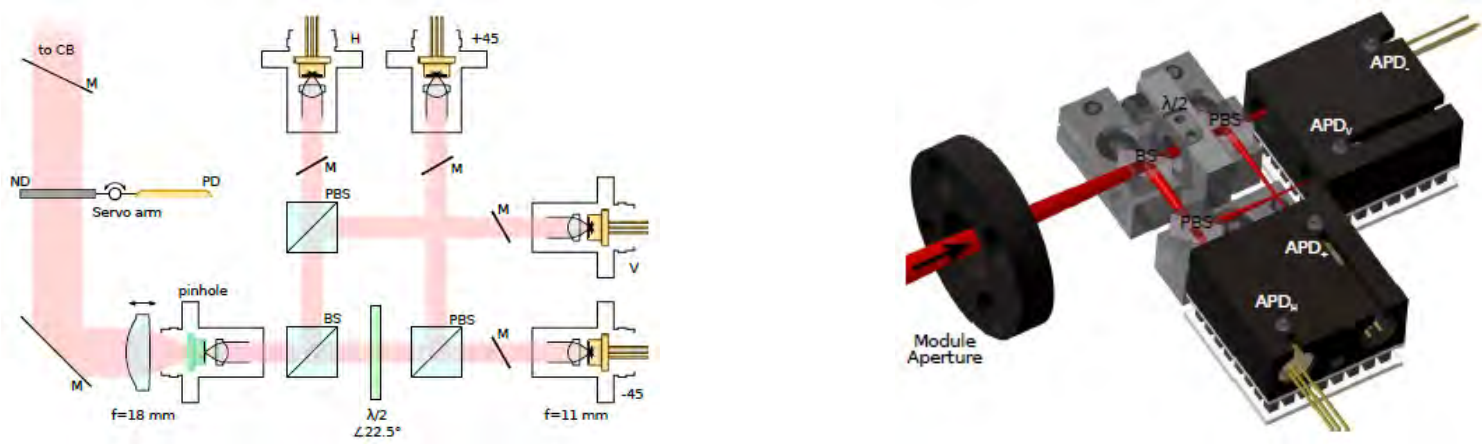

Figure 1: (left) Sketch of the sender module showing 4 laserdiodes with outcouplers, together with polarizing (PBS) and polarization independent (BS) beamsplitters and a $\lambda / 2$-plate rotating the polarization in order to combine and to define the polarization of light emitted by the four diodes. (right) Engineering drawing of the receiver employing similar optics to split incoming signal to the avalanche photo diodes (APD) registering photons in the respective polarization state.

The receiver module (Bob) is constructed similarly using BS, half-wave-plate and PBS to analyze the polarization of the incoming signal randomly in either the $\mathrm{H} / \mathrm{V}$ or the $+45^{\circ} / 45^{\circ}$ basis. Both modules were equipped with custom designed flexure stages to enable alignment while providing stability against temperature changes and vibrations. In addition, at the receiver side, a fully automatized polarization alignment was necessary in order to account for tilts of the airplane or of the receiving telescope, respectively. This system consisting of three waveplates was calibrated on the respective position data obtained live from the airplane warranting good overlap of Alice' and Bob's polarization bases. Both QKDmodules where kept with small footprint to accommodate them in the already existing classical communication setup. The system is driven at a $10 \mathrm{MHz}$ clock rate with pulse duration of about $1 \mathrm{~ns}$ and detection windows as small as $2 \mathrm{~ns}$ to reduce noise from dark counts and straylight.

\subsection{FSO system}

The FSO system enables Alice and Bob to set up the optical link between aircraft and ground. Once the optical link is acquired, QKD experiments can start. The FSO communication beam is used as beacon source and to transmit a synchronization signal. Alice was installed on aircraft side. The aircraft equipment as originally developed for FSO communications downlinks [18] is modified and used as airborne experimental platform. The Alice module is integrated without interfering the original communications capabilities. Bob is installed on ground. Its host system is the Optical Ground Station Oberpfaffenhofen as developed for satellite, aircraft and stratosphere to ground communications and channel measurements [18], [23-25]. A pointing, acquisition and tracking process (PAT) covers all necessary steps to obtain and maintain line of sight between Alice and Bob. The sync signal transmission is used to temporally align transmission and reception of the weak coherent pulses used in the QKD scheme. The functional block diagram of the FSO system with integrated QKD part is shown in Figure 2. The left block describes the sender terminal which is installed in a Do228 aircraft. The terminal optical bench is mounted onto the seat rails in the aircraft cabin. The laser beam is guided via a coudé system through a hole in the fuselage to the outside of the aircraft. Only the optical head protected by an optical glass dome is exposed to the air stream (Figure 3, left). The right block describes the optical ground station. It was located on the rooftop of the Institute of Communications and Navigation at DLR site Oberpfaffenhofen, near Munich, Germany. The receiver telescope is steered by a fork mount construction installed in an astronomical dome (Figure 3, right). The PAT process for optical link set up works as follows: the first step is a telescope pointing based on GPS location data from the aircraft and the ground station. The GPS data of the ground station is fix, the data from the aircraft is sent to ground via a low rate UHF link. The second step is illumination of the aircraft from ground with a beacon laser system with large beam divergence aligned to the ground telescope. The laser signal is detected by a position sensitive device in the aircraft terminal. This way, the aircraft terminal obtains most precise information on the ground station location and increases pointing accuracy of the sensor and the downlink beam. In the third step, the ground station senses the aircraft signal and also optimizes beam pointing. Eventually, line of sight 
is obtained between transmitter and receiver and the link is acquired. In the fourth step, both sides continue optical tracking.

The optical system of the aircraft terminal and the ground station comprise two optical paths, respectively: the FSO and QKD path. The FSO downlink/uplink path is at 1550/1590 nm, the QKD path at $850 \mathrm{~nm}$ wavelength. The FSO components in Figure 2 are depicted with black lined boxes, the QKD components with green lined boxes. The FSO signal from the ground laser beacon (red arrow) is received by the aircraft $\mathrm{Rx}$-Tx telescope steered by the coarse pointing assembly (CPA). The CPA comprises two high precision direct drive motors. The main terminal computer controls the motors with inputs from the IMU (Inertial Measurement Unit) and the Wide Field of View (WFoW) camera as the position sensitive device. The beam is stabilized by the fine pointing assembly (FPA), a fast voice coil actuated mirror controlled with a digital signal processor (DSP) and signals from a four-quadrant diode (4QD). A dichroic mirror transmits the FSO signal to a coupling system that distributes the beam to the WFoV camera and the 4QD. The FSO Txbeam takes till the coupling system the same optical path as the Rx-beam. The laser diode creates a $100 \mathrm{MHz}$ modulated signal (on-off-keying) which is amplified by the erbium doped fiber amplifier (EDFA). The QKD-beam is fed via the reflection path of the dichroic mirror. Weak coherent pulses are sent with $10 \mathrm{MHz}$ frequency. The FSO and QKD signals are received by the ground station telescope. A WFoV camera gives input signals to the mount control which is aligned aside the main telescope. Another FPA, a fast piezo actuated mirror, stabilizes the beam. The FPA is controlled with signals from the Narrow Field of View (NFoV) camera. The receiver front-end (RFE) detects the $100 \mathrm{MHz}$ sync signal. The coupling system distributes the signals between the RFE and the NFoV camera. The QKD receiver is on the reflection side of the dichroic mirror. A polarization controller compensates the rotation of polarization plane due to the aircraft terminal beam control. Eventually, the beam is fed to the BB84 analyzer. The beacon laser system, comprising two fiber lasers and two 2" collimators, is aligned aside the main telescope.

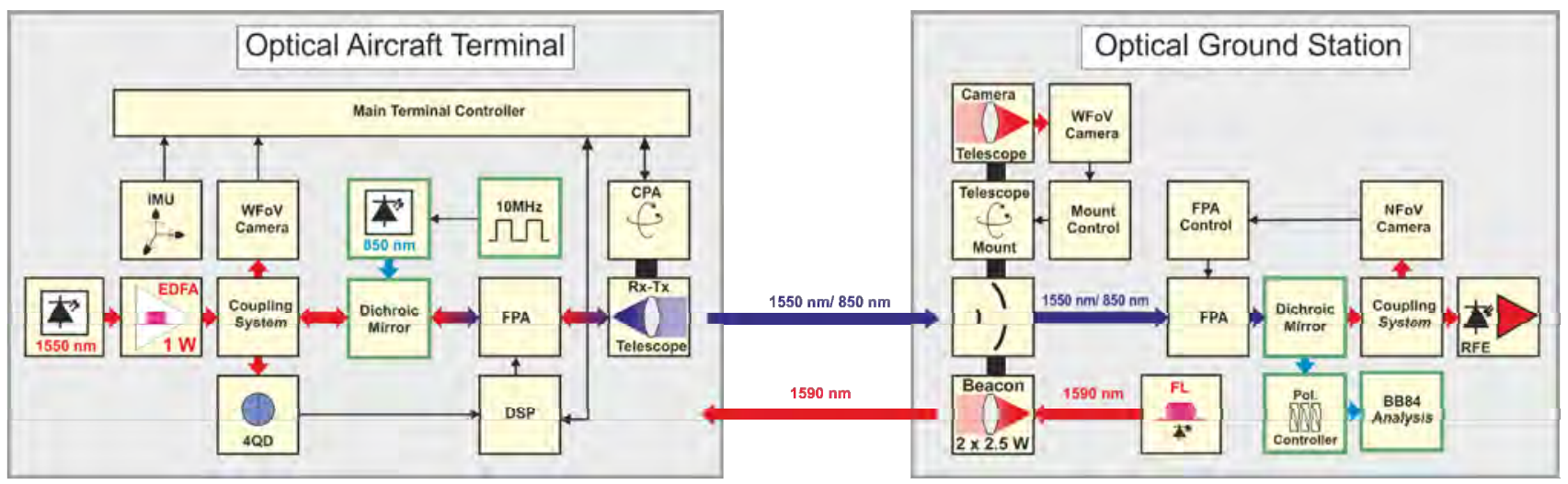

Figure 2: Functional block diagram of FSO and QKD link system. The communications signal part is denoted with black lined boxes, the QKD signal part with green lined boxes.

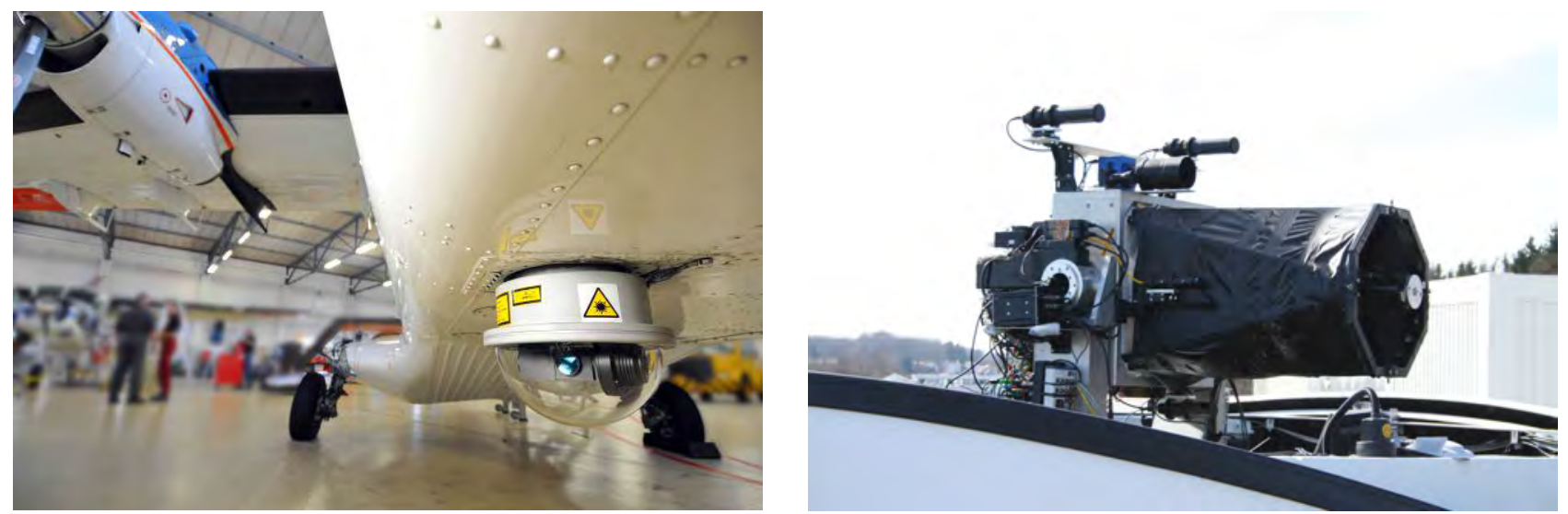

Figure 3: (left) Optical head of aircraft terminal and (right) receiver telescope of optical ground station. Source: DLR. 
A detailed link budget analysis was performed to estimate the expected coupling efficiency and to plan the experiment geometry, i.e. aircraft height and distance [21].

\section{FREE-SPACE OPTICAL SATELLITE COMMUNICATIONS DEVELOPMENTS}

\subsection{Satellite terminals}

Together with the increasing resolution of earth observation sensor systems on small LEO satellites, the demand for high data rate transmission from LEO orbit direct to Earth increases accordingly. In the project OSIRIS, DLR developed highly compact optical communication terminals for small LEO satellites. The first generation of OSIRIS provides a 200 Mbps link for the Flying Laptop satellite of University of Stuttgart. The Flying Laptop is a $120 \mathrm{~kg}$ satellite with dimensions of $80 \times 60$ x $50 \mathrm{~cm}$ and a Multispectral Imaging Camera System (MICS) as primary payload. Figure 4 shows an image of the satellite with integrated OSIRIS payload. With a ground sample distance of $25 \mathrm{~m}$ and a swath width of $25 \mathrm{~km}$ of the camera, the Flying Laptop is additionally equipped with the optical communication terminal as secondary payload. The pointing of the laser beam of OSIRIS onboard is based on the attitude control system of the satellite bus. Based on the star sensors, the attitude control points the laser beam in an open loop control circuit to the optical ground station. The beam divergence of the transmission beam is adapted to the attitude control accuracy and limits the data rate to $200 \mathrm{Mbps}$. OSIRIS comes with a system weight of $1,3 \mathrm{~kg}$ and a maximum power consumption of $26 \mathrm{~W}$. Figure 4 (left) shows the satellite during preparation for the thermal-vacuum testing.


Figure 4: (left) Flying Laptop satellite with integrated OSIRIS payload. The 2 x $10 \mathrm{~mm}$ refractor telescopes are behind the black beam dump which is removed before flight. Source: University Stuttgart. (right) OSIRIS Flight Model for the BiROS Satellite. Source: DLR

The second generation of OSIRIS has been developed for DLR's BiROS satellite, designed and integrated at DLR's Institute of Optical Sensor System in Berlin. BiROS is designed with dimensions of 58 x 88 x $68 \mathrm{~cm}$ and a weight of 130 $\mathrm{kg}$ at which $60 \mathrm{~kg}$ are reserved for payloads. The primary payload consits of an earth observation camera system with 2 infrarad channels and one in the visible spectrum. The development of the OSIRIS payload has been based on the laser source also used for the Flying Laptop. For the BiROS satellite, the OSIRIS system is additionally equipped with a tracking sensor which allows for an additional input into the attitute control system of the satellite which performs the pointing of the laser beam. With this additional sensor, the attitude control accuracy can be increased and allows for a reduction of the beam divergence of the transmission laser beam - a data rate of $1 \mathrm{Gbit} / \mathrm{s}$ can be realized for LEO-ground links from BiROS. With the tracking sensor, OSIRIS is also able to provide an optical uplink channel to the satellite with a maximum data rate of $1 \mathrm{Mbit} / \mathrm{s}$. Figure 4 (right) shows the Flight Models of OSIRIS for the BiROS satellite with the optical bench (right), laser source box (middle) and receiver electronics box (left). The Engineering and Qualification Models underwent a space qualification following established standards for vibration, thermal-vacuum as well as Total Ionizing Dose testing. Since all OSIRIS developments are currently based on COTS (Commercial Of-the Shelf) components, the space qualification allows for a highly compact as well as highly efficient system design.

Currently, the development of a $3^{\text {rd }}$-generation OSIRIS-terminal is under preparation, called OSIRIS-V3. It builds on the heritage of the previous OSIRIS developments, and will contain a coarse pointing assembly to enable satelliteindependent beam pointing. The performance aims are $5 \mathrm{~kg}$ weight, $50 \mathrm{~W}$ power consumption as well as $10 \mathrm{Gbps}$ data 
rate. The development aims on small LEO satellites as well, with the goal of supplying a powerful data link also for this satellite class. Figure 5 (left) shows the proposed conceptual design of the system.

Besides the traditional satellite classes in the $100 \mathrm{~kg}$ domain, also smaller platforms in the range of a few kilograms become more and more mature in the field of earth observation missions. Increasing sensor resolutions and required payloads also drive the development of high data rate communication systems on CubeSats. CubeSats add additional requirements to the system design since size, weight and power are very limiting factors on these platforms as well as reduced costs and development times. Figure 5 (right) shows a CAD model of the OSIRIS payload for CubeSats. The system provides a data rate adapted to the satellite bus speeds of $100 \mathrm{Mbps}$ at a system weight of 300 grams and a maximum power consumption of $7 \mathrm{~W}$ during the downlink. As shown in the figure, the system occupies around 1/3 of a one unit (1U) CubeSat. To reduce the requirements for the attitude control system of the CubeSat bus, OSIRIS is equipped with a Fine Pointing Assembly (FPA) which compensates misalignments of the satellite bus against the ground station of a few degrees. As well as the other OSIRIS generations, also this version is based on COTS components. Main components like tracking sensor and laser source have already been qualified in the first and second generation of OSIRIS on Flying Laptop and BiROS and allow for a low risk system design for the CubeSat optical payload.
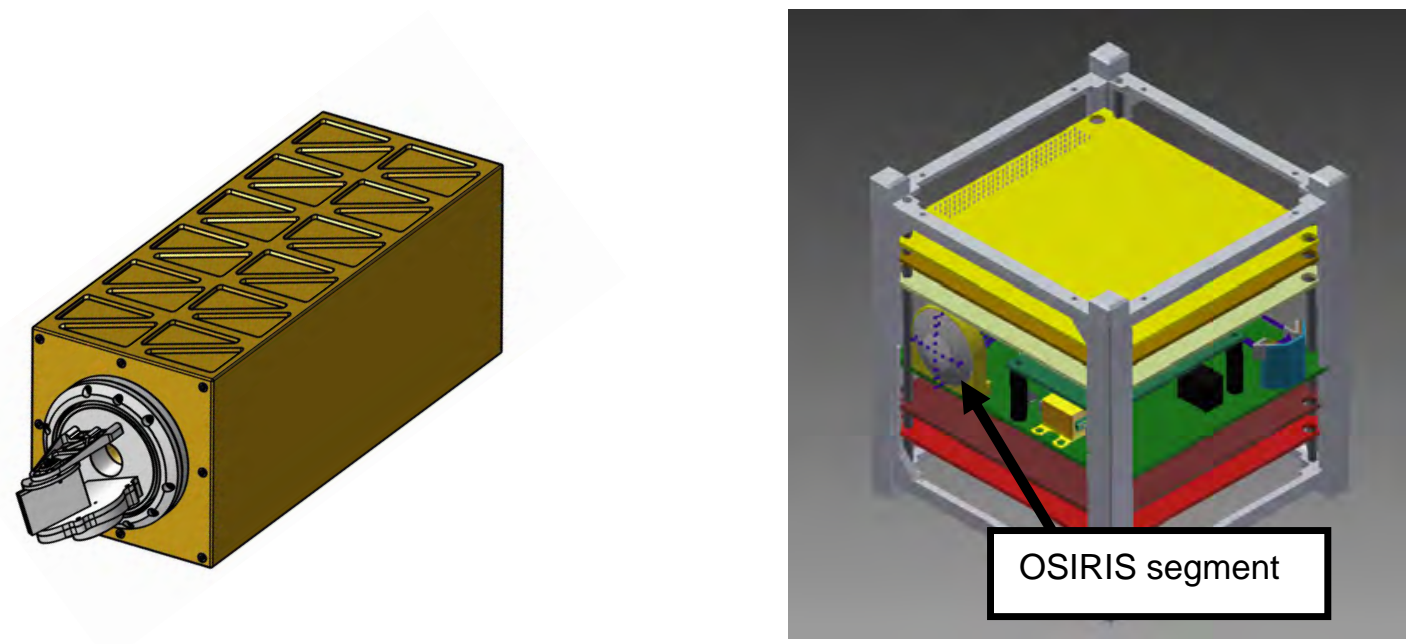

Figure 5: (left) Preliminary design study of a miniaturized OSIRIS payload for a 1Unit CubeSat. (right) Conceptual design of third generation OSIRIS terminal optimized for downlinks from small LEO satellites.

\subsection{Ground stations}

The DLR Institute of Communications and Navigation operates two optical ground stations, the Optical Ground Station Oberpfaffenhofen (OGS-OP) and the Transportable Optical Ground Station. The OGS-OP (Figure 6, left) is a fix installation on the institutes building rooftop. It serves as an experimental station and laboratory for various kinds of experiments. The pre-version of the OGS-OP was used for the airborne QKD-experiment as described in [9], [21]. Further use of the OGS-OP are channel measurement and communications tests for satellite downlinks as done in the framework of the KIODO project [24], [25] and currently in the experiments with the OPALS system onboard the International Space Station [26]. Furthermore, measurement campaigns were also undertaken for channel characterization in the air-ground scenario [27].

The Transportable Optical Ground Station (Figure 6, right) has a higher degree of integration and is therefore meant for semi-operational use and technology demonstrations, e.g. for satellite- and aircraft-ground communications [19], [2830]. 

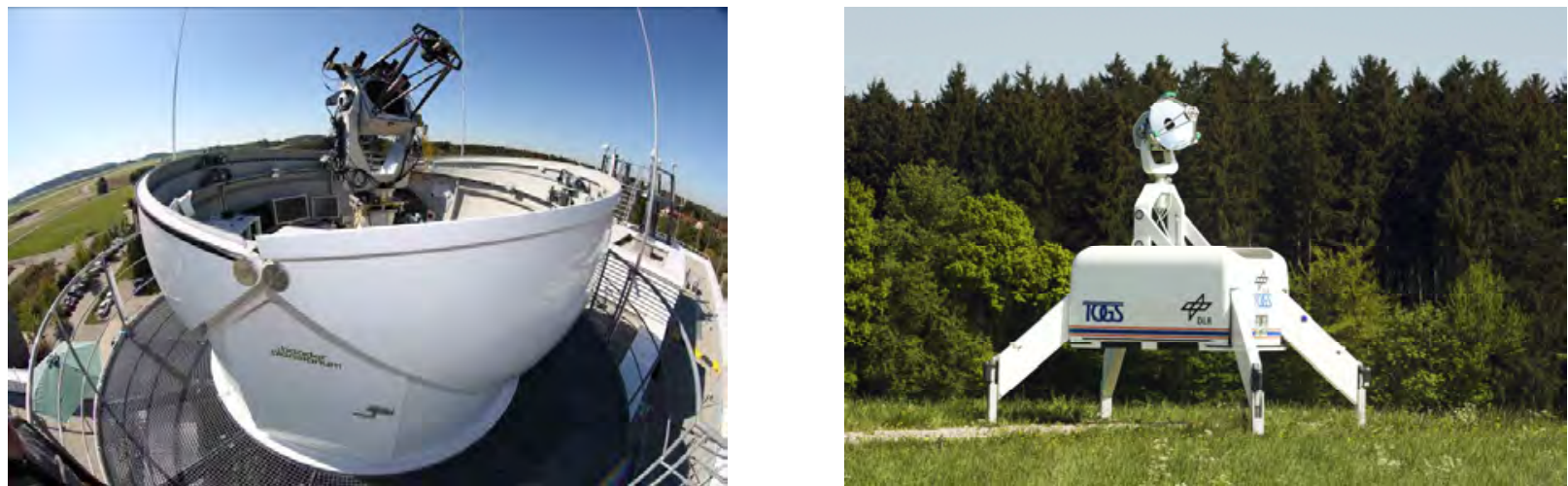

Figure 6: (left) Optical Ground Station Oberpfaffenhofen and (right) Transportable Optical Ground Station. Source: DLR.

\section{QUANTUM KEY DISTRIBUTION DEVELOPMENTS: MINIATURIZATION OF ALICE}

The footprint of the first flight-module of $80 \times 100 \times 150 \mathrm{~mm}^{3}$ is surely too big for a CubeSat. However, using micro optics and new developments in photonic waveguides enabled a new level of integration of the transmitter module optics [31]. Again, the polarization qubits $\left\{\mathrm{H}, \mathrm{V},+45^{\circ},-45^{\circ}\right\}$ are faint near infrared laser pulses, now generated at $100 \mathrm{MHz}$ repetition rate. The light pulses are generated by four Vertical Cavity Surface Emitting Lasers (VCSEL) fabricated as array with a separation of the four diodes by only $250 \mu \mathrm{m}$ each (Figure 7). They exhibit highly similar emission properties and produce 100 ps pulses. There is no option for rotating them to obtain the different polarization directions, however, for such short pulse duration, the degree of polarization turned out to be rather low. We thus employ additional polarizers which are now oriented according the requirements of the BB84 protocol. For this purpose, four wire-grid polarizers fabricated using focused ion beam milling where produced. With improved modelling structure optimization yields polarizers exhibiting extinction ratios up to $29 \mathrm{~dB}$ [32] enabling high fidelity control of the qubit state associated with each diode. The intensity and time arrival of the pulses can be finely tuned to obtain perfect temporal overlap between the four channels. To ensure the spatial indistinguishability of the states, the four polarized beams are coupled into a low-birefringence, single-mode waveguide array manufactured via femtosecond laser micromachining in a borosilicate glass chip. The 3D capability of the fabrication technique was exploited to minimize polarization dependent effects in the directional couplers. Full process tomography was performed to optimize the input states and ensure QBERs below $1 \%$.
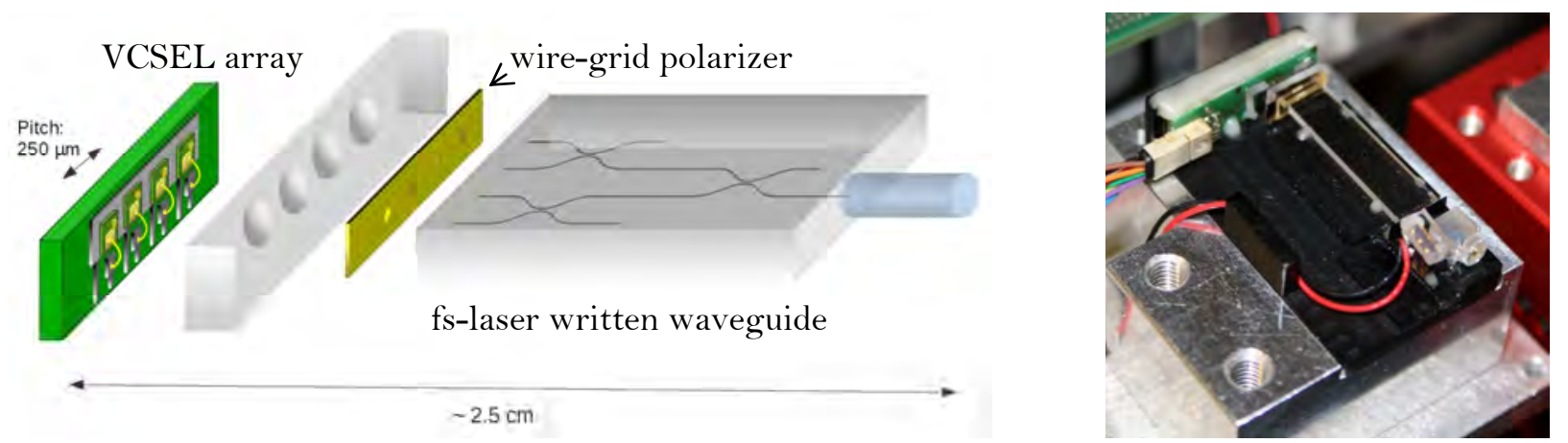

Figure 7: (left) Scheme of fully integrated QKD-sender optics employing VCSEL array (green) with pitch 250 $\mu \mathrm{m}$, wire-grid polarizer (yellow) followed by a femto-second laser written waveguide array for overlapping signals from the four diodes. (right) Photograph of the assembled module.

\section{OUTLOOK}

Former work of DLR and LMU in the field of aeronautical QKD experiments is presented. This work is a precursor experiment to future QKD satellite systems. The lessons learned from the air-ground experiment give a list of necessary 
issues to work on for realization of a QKD-satellite system. These are, amongst others, the increase of coupling efficiency, further miniaturization of the QKD transmitter and receiver, space qualification of QKD transmitter, filtering techniques to enable daylight functionality, as well as more sensitive single photon receivers. Current developments of DLR both on space segment and ground station side are presented and may be exploited to be used in future QKD satellite systems. Both generations of the space segment (OSIRIS) are based on the attitude control system of the satellite bus. In the next development generation "OSIRIS-V3", the payload will be additionally equipped with a high precision coarse pointing assembly. This will enable higher pointing accuracies, narrower beam divergences and therefore higher coupling efficiencies. A smaller version of an optical communication terminal shall be developed for use on CubeSat systems. For the possibility of special measurements setups, the OGS will be extended to feature a coudé room. This will enable easy installation of interferometric measurement devices, adaptive optics and more complex receiver systems.

Miniaturization development of Alice is described. The new module is significantly smaller than the preceding version. On the receiver side, future work has to focus on utilizing the improved pointing accuracy for much narrower spatial filtering. In addition, fast detectors will be required to reduce the detection time windows to clearly below $1 \mathrm{~ns}$. Additional spectral filtering will further reduce background noise to enable key exchange also in case of low link efficiency.

\section{REFERENCES}

[1] B. Korzh, C. C. W. Lim, R. Houlmann, N. Gisin, M. J. Li, D. Nolan, B. Sanguinetti, R. Thew, and H. Zbinden, "Provably secure and practical quantum key distribution over 307?km of optical fibre," Nature Photonics, vol. 9, pp. 163-168, 2015.

[2] J. M. P. Armengol, B. Furch, C. J. de Matos, O. Minster, L. Cacciapuoti, M. Pfennigbauer, M. A. T. Jennewein, R. Ursin, T. Schmitt-Manderbach, G. Baister, J. Rarity, W. Leeb, C. Barbieri, HaraldWeinfurter, and A. Zeilinger, "Quantum communications at ESA: Towards a space experiment on the ISS," Acta Astronautica, vol. 63, pp. 165-178, 2008.

[3] R. J. Hughes, J. E. Nordholt, K. P. McCabe, R. T. Newell, and C. G. Peterson, "Satellite-based qunatum communications," in Proceedings of Updating Quantum Cryptography and Communications 2010, 2010, pp. 7172.

[4] T. Jennewein, J. P. Bourgoin, B. Higgins, C. Holloway, E. Meyer-Scott, C. Erven, B. Heim, Z. Yan, H. Hübel, G. Weihs, E. Choi, I. D’Souza, D. Hudson, and R. Laflamme, “QEYSSAT: a mission proposal for a quantum receiver in space," in Proceedings of SPIE 8997, 2014.

[5] T. Jennewein, C. Grant, E. Choi, C. Pugh, C. Holloway, J. Bourgoin, H. Hakima, B. Higgins, and R. Zee, "The NanoQEY Mission: Ground to Space Quantum Key and Entanglement Distribution Using a Nanosatellite,” in Proceedings of SPIE Quantum-Physics-Based Information Security Conference, 2014.

[6] J. E. Nordholt, R. J. Hughes, G. Morgan, C. G. Peterson, and C. C. Wipf, "Present and Future Free-Space Quantum Key Distribution,” in Proceedings of SPIE 4635, 2002.

[7] I. D. Souza, D. Hudson, C. Evans, E. Choi, and T. J. J. Sarda, "The QEYSSat Mission: Demonstrating Global Quantum Key Distribution Using a Microsatellite," in Proceedings of 26th Annual AIAA/USU Conference on Small Satellites, 2012.

[8] D. Elser, K. Günthner, I. Khan, B. Stiller, C. Marquardt, G. Leuchs, K. Saucke, D. Tröndle, F. Heine, S. Seel, P. Greulich, H. Zech, B. Gütlich, I. Richter, and R. Meyer, "Satellite Quantum Communication Via the Alphasat Laser Communication Terminal," in Proceedings of ICSOS 2015, 2015.

[9] S. Nauerth, F. Moll, M. Rau, C. Fuchs, J. Horwath, S. Frick, and H. Weinfurter, "Air-to-ground quantum communication,” Nature Photonics, vol. 7, pp. 382-386, 2013.

[10] P. Villoresi, T. Jennewein, F. Tamburini, M. Aspelmeyer, C. Bonato, R. Ursin, C. Pernechele, V. Luceri, G. Bianco, A. Zeilinger, and and C. Barbieri, "Experimental verification of the feasibility of a quantum channel between space and Earth," New Journal of Physics, vol. 10, 2008.

[11] J.-Y. Wang, B. Yang, S.-K. Liao, L. Zhang, Q. Shen, X.-F. Hu, J.-C. Wu, S.-J. Yang, H. Jiang, Y.-L. Tang, B. Zhong, H. Liang, W.-Y. Liu, Y.-H. Hu, Y.-M. Huang, B. Qi, J.-G. Ren, G.-S. Pan, J. Yin, J.-J. Jia, Y.-A. Chen, K. Chen, C.-Z. Peng, and J.-W. Pan, "Direct and full-scale experimental verifications towards ground-satellite quantum key distribution,” Nature Photonics, vol. 7, pp. 387-393, 2013. 
[12] J. Yin, Y. Cao, S.-B. Liu, G.-S. Pan, Jin-Hong, Wang, T. Yang, Z.-P. Zhang, F.-M. Yang, Y.-A. Chen, C.-Z. Peng, and J.-W. Pan, "Experimental quasi-single-photon transmission from satellite to earth," OPTICS EXPRESS, vol. 21, pp. 20032-20040, 2013.

[13] C. Schmidt, M. Brechtelsbauer, F. Rein, and C. Fuchs, “OSIRIS payload for DLR's BiROS satellite,” in International Conference on Space Optical Systems and Applications 2014, 2014.

[14] H. Hauschildt, F. Garat, H. Greus, K. Kably, J.-P. Lejault, H. L. Moeller, A. Murrell, J. Perdigues, D. M. Witting, B. Theelen, M. Wiegand, and A. Hegyi, "European Data Relay System - one year to go!,” in Proc. International Conference on Space Optical Systems and Applications (ICSOS) 2014, 2014.

[15] D. M. Boroson, "Overview of the Lunar Laser Communication Demonstration," in Proc. International Conference on Space Optical Systems and Applications (ICSOS) 2014, 2014.

[16] J. Poliak, D. Giggenbach, F. Moll, F. Rein, C. Fuchs, and R. M. Calvo, “Terabit-Throughput GEO Satellite Optical Feeder Link Testbed," in Proceedings of 13th ConTEL, 2015.

[17] V. Cazaubiel, G. Planche, V. Chorvalli, L. Le Hors, B. Roy, and E. Giraud, "LOLA: A 40000 km Optical Link between an Aircraft and a Geostationary Satellite,” in Sixth International Conference on Space Optics, 2006, p. 87.1.

[18] J. Horwath and C. Fuchs, "Aircraft to ground unidirectional laser-communications terminal for high-resolution sensors,” in Proc. SPIE, Free-Space Laser Communication Technologies XXI, 2009, vol. 7199.

[19] F. Moll, J. Horwath, A. Shrestha, M. Brechtelsbauer, C. Fuchs, L. A. M. Navajas, A. M. L. Souto, and D. D. González, "Demonstration of High-Rate Laser Communications From a Fast Airborne Platform,” IEEE JOURNAL ON SELECTED AREAS IN COMMUNICATIONS, vol. 33, pp. 1985-1995, 2015.

[20] K.-D. Büchter and A. Sizmann, "High-bandwidth Aeronautical Telecommunication Options," in Deutscher Luftund Raumfahrtkongress 2014 (accepted), 2014.

[21] F. Moll, S. Nauerth, C. Fuchs, J. Horwath, M. Rau, and H. Weinfurter, "Communication system technology for demonstration of BB84 quantum key distribution in optical aircraft downlinks," in Proc. SPIE 8517 (Laser Communication and Propagation through the Atmosphere and Oceans), 2012, vol. 8517.

[22] N. Gisin, G. Ribordy, W. Tittel, and H. Zbinden, “Quantum cryptography,” Rev. Mod. Phys., vol. 74, pp. 145195, 2002.

[23] J. Horwath, N. Perlot, M. Knapek, and F. Moll, "Experimental verification of optical backhaul links for highaltitude platform networks: Atmospheric turbulence and downlink availability,” Int. J. Satell. Commun. Network, vol. 25, pp. 501-528, 2007.

[24] F. Moll, "KIODO 2009: Trials and analysis," in GOLCE 2010, 2010.

[25] N. Perlot, M. Knapek, D. Giggenbach, J. Horwath, M. Brechtelsbauer, Y. Takayama, and T. Jono, "Results of the optical downlink experiment KIODO from OICETS satellite to Optical Ground Station Oberpfaffenhofen (OGSOP),” in Free-Space Laser Communication Technologies XIX and Atmospheric Propagation of Electromagnetic Waves, 2007, vol. 6457, p. 645704.

[26] B. V. Oaida, M. J. Abrahamson, R. J. Witoff, J. N. B. Martinez, and D. A. Zayas, "OPALS: an optical communications technology demonstration from the International Space Station,” in IEEE, 2013, pp. 1-20.

[27] K. Shortt, D. Giggenbach, R. Mata-Calvo, F. Moll, C. Fuchs, C. Schmidt, J. Horwath, J. Yeh, V. Selvaraj, and R. Banerjee, "Channel characterization for air-to-ground free-space optical communication links," in Proc. SPIE 8971 (Free-Space Laser Communication and Atmospheric Propagation XXVI), 2014, vol. 8971.

[28] F. Moll, A. Shrestha, and C. Fuchs, "Ground stations for aeronautical and space laser communications at German Aerospace Center,” in Proc. of SPIE 9647, 2015, no. 9647.

[29] C. Schmidt, J. Horwath, A. Shrestha, F. Moll, M. Brechtelsbauer, and C. Fuchs, "High-speed, high-volume optical communication for aircraft," SPIE Newsroom. 2013.

[30] A. Shrestha and M. Brechtelsbauer, "Transportable optical ground station for high-speed free-space laser communication," in Proc. SPIE 8517 (Laser Communication and Propagation through the Atmosphere and Oceans), 2012, vol. 8517.

[31] G. Vest, M. Rau, L. Fuchs, G. Corrielli, H. Weier, S. Nauerth, A. Crespi, R. Osellame, and H. Weinfurter, "Design and evaluation of a handheld quantum key distribution sender module," 2015.

[32] G. Mélen, W. Rosenfeld, and H. Weinfurter, "Impact of the slit geometry on the performance of wire-grid polarisers,” Optics Express, vol. 23, pp. 32171-32178, 2015. 